\title{
Tuning of synapse number, structure and function in the cochlea
}

\author{
Alexander C Meyer ${ }^{1,6}$, Thomas Frank ${ }^{1,6}$, Darina Khimich ${ }^{1,6}$, Gerhard Hoch ${ }^{1}$, Dietmar Riedel ${ }^{2}$, \\ Nikolai M Chapochnikov ${ }^{1,3}$, Yury M Yarin ${ }^{1,4}$, Benjamin Harke ${ }^{5}$, Stefan W Hell ${ }^{5}$, \\ Alexander Egner ${ }^{5}$ \& Tobias Moser ${ }^{1,3}$
}

\begin{abstract}
Cochlear inner hair cells (IHCs) transmit acoustic information to spiral ganglion neurons through ribbon synapses. Here we have used morphological and physiological techniques to ask whether synaptic mechanisms differ along the tonotopic axis and within IHCs in the mouse cochlea. We show that the number of ribbon synapses per IHC peaks where the cochlea is most sensitive to sound. Exocytosis, measured as membrane capacitance changes, scaled with synapse number when comparing apical and midcochlear IHCs. Synapses were distributed in the subnuclear portion of IHCs. High-resolution imaging of IHC synapses provided insights into presynaptic $\mathrm{Ca}^{2+}$ channel clusters and $\mathrm{Ca}^{2+}$ signals, synaptic ribbons and postsynaptic glutamate receptor clusters and revealed subtle differences in their average properties along the tonotopic axis. However, we observed substantial variability for presynaptic $\mathrm{Ca}^{2+}$ signals, even within individual IHCs, providing a candidate presynaptic mechanism for the divergent dynamics of spiral ganglion neuron spiking.
\end{abstract}

After processing by the mammalian ear's exquisite micromechanics and mechanoelectrical transduction, acoustic information is encoded at the afferent synapses of IHCs with high temporal precision ${ }^{1,2}$. Presynaptic active zones of IHCs contain a synaptic ribbon, a multiprotein structure that tethers synaptic vesicles ${ }^{3,4}$ and ensures a large pool of readily releasable vesicles ${ }^{5-8}$. Stimulus-secretion coupling is governed by $\mathrm{Ca}_{V} 1.3 \mathrm{~L}$-type $\mathrm{Ca}^{2+}$ channels ${ }^{9,10}$ that tightly control the release of glutamate from nearby fusion-competent vesicles ${ }^{2,8,11}$ onto postsynaptic AMPA receptors ${ }^{12,13}$ on the unbranched peripheral axon of the bipolar spiral ganglion neuron $(\mathrm{SGN})^{14}$. As a result, each SGN receives input from only one IHC active zone, while each IHC drives several SGNs.

Whether and how the number and properties of afferent synapses of the cochlea are 'tuned' for optimal sound encoding remains an important question (refs. 15,16, for example). The frequency selectivity of SGNs is primarily determined by the location of the innervated IHC on the cochlea's tonotopic axis, providing a place code for frequency (refs. 17,18, for example). In addition, studies of small samples of synapses from distinct cochlear regions have indicated that the innervation density varies along the length of the cochlea ${ }^{19-21}$. Moreover, it has been shown that SGNs covering a narrow frequency range differ markedly in spontaneous and evoked firing rates, sound threshold and dynamic range (for example, refs. 22,23) and that they collectively encode a large range of sound pressures. It is generally believed, but not yet directly proven, that each IHC makes contact with such physiologically diverse SGNs. If true, the heterogeneity of SGN dynamics could be caused by pre- and postsynaptic mechanisms $7,19,24,25$. Pioneering work on the cat cochlea suggested that lowspontaneous-rate SGNs preferentially contact active zones with large or even multiple synaptic ribbons at the neural side of IHCs (toward the modiolus), whereas high spontaneous rate SGNs are driven by small, 'simple' synapses at the abneural IHC side (toward the outer hair cells ${ }^{24}$.

Here we have used patch-clamp, confocal imaging of IHC presynaptic $\mathrm{Ca}^{2+}$ signals; confocal, $4 \mathrm{Pi}^{26,27}$ and stimulated emission depletion (STED) ${ }^{28}$ microscopy of immunolabeled synapses; and electron microscopy to characterize the distribution of afferent synapses as well as their structure and function at different tonotopic regions of the cochlea. Having investigated thousands of synapses in hundreds of IHCs, we provide a continuous representation of synapse number per IHC along the entire mouse cochlea, and we show that synapse density parallels the neuronal population audiogram. Using STED microscopy, we provide optical, nanometer-scale measurements of individual clusters of presynaptic $\mathrm{Ca}^{2+}$ channels and postsynaptic AMPA receptors. Whereas average structural and functional synapse properties varied only slightly along the cochlea's tonotopic axis, we found considerable heterogeneity of presynaptic $\mathrm{Ca}^{2+}$ signals among the synapses in IHCs in a given region.

${ }^{1}$ InnerEarLab, Department of Otolaryngology and Center for Molecular Physiology of the Brain, University of Göttingen, Göttingen, Germany. ${ }^{2}$ Laboratory of Electron Microscopy, Max-Planck-Institute for Biophysical Chemistry, Göttingen, Germany. ${ }^{3}$ Bernstein Center for Computational Neuroscience, University of Göttingen, Göttingen, Germany. ${ }^{4}$ Clinic of Otorhinolaryngology, Department of Medicine, Technical University of Dresden, Dresden, Germany. ${ }^{5}$ Department of NanoBiophotonics, Max-PlanckInstitute for Biophysical Chemistry, Göttingen, Germany. ${ }^{6}$ These authors contributed equally to the work. Correspondence should be addressed to T.M. (tmoser@gwdg.de) or A.E. (aegner@gwdg.de). 
Figure 1 The number of afferent synapses per IHC co-varies with ABR threshold along the tonotopic axis. (a) Projections of confocal stacks of immunostained mouse IHC afferent synapses at different tonotopic locations (red, anti-CtBP2/ RIBEYE; green, anti-GluR2/3; blue, Hoechst 34580 nuclear stain). Percentages indicate the normalized location of the imaged IHCs in the cochlea (0\%, apical end; $100 \%$, basal end). Center: montage of the low-magnification view of fragments of the full explanted organ of Corti. Red circles, locations of the confocal images. (b) Synaptic cochleograms and tone-burst ABR audiograms of NMRI and C57BL/6 mice (together, more than 15,000 synapses) overlaid by relating distance to cochlear apex to the tonotopic map of mice of strain CBA (top axis ${ }^{18}$ ). NMRI mice: red open circles, number of synapses per IHC (26 ears, P15-P20); red filled circles, $A B R$ threshold (average \pm s.e.m., ten ears from six mice). C57BL/6: black open squares, number of synapses per IHC (two ears, P30); connected black filled squares, ABR threshold. Continuous black line: fit of a quadratic function to the collective data set of both mouse lines.

(c) Synaptic cochleograms (circles): number of synapses per IHC (more than 10,000 synapses in six ears from four P28 gerbils), plotted against a tonotopic map (taken from ref. 29) and frequencydependent compound action potentials (CAP) thresholds (from ref. 29) (red line). Continuous black line: fit of a quadratic function to the data.
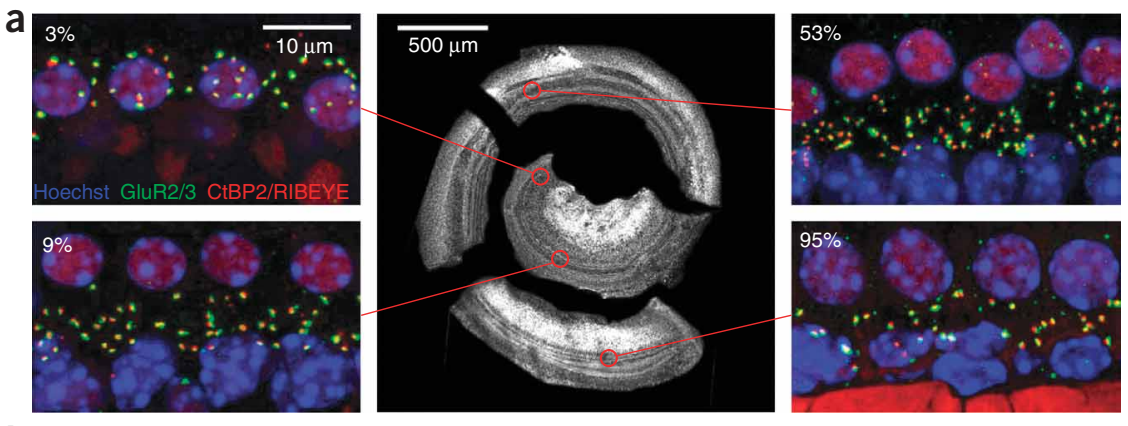

b Mouse

$$
\text { C Gerbil }
$$

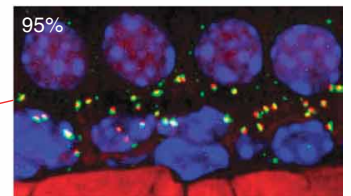

Frequency
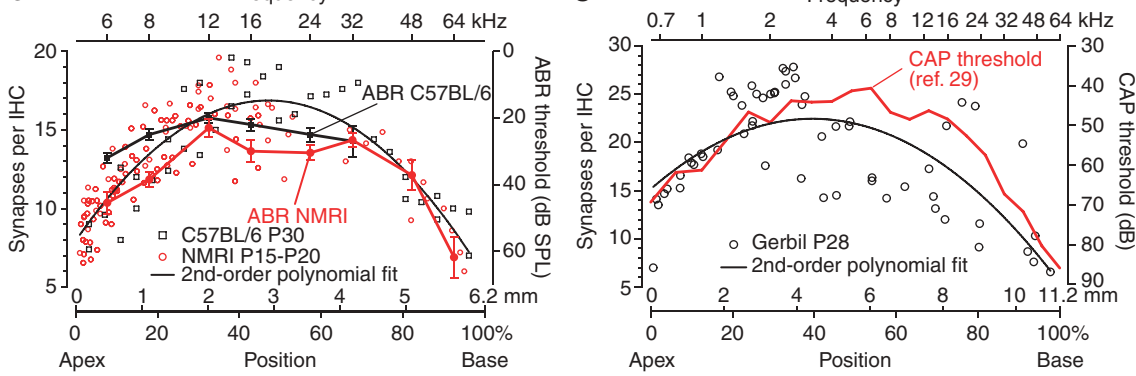

\section{RESULTS}

\section{Tonotopic synapse density map and subcellular distribution}

First, we assessed the number of ribbon synapses per IHC throughout the entire cochlea (creating a synaptic cochleogram) of mice (Fig. 1a,b; high-frequency hearing) and gerbils (Fig. 1c and Supplementary Fig. 1 online; low-frequency hearing) by confocal microscopy of immunolabeled, whole-mount organs of Corti. The microdissected parts of the organ of Corti were aligned for measuring the distance of a given synapse to the apex of the cochlea (see Methods; Fig. 1a). We identified IHC ribbon synapses as colocalized spots of presynaptic ribbons (using antibody to transcription factor CtBP2 sharing homologous domain with RIBEYE, the main protein component of the synaptic ribbon) and postsynaptic GluR2/3 (labeling the AMPA receptor clusters) immunofluorescence in stacks of confocal sections, and divided their count by the number of IHC nuclei ${ }^{6}$. The study used hearing mice (as determined by auditory brainstem responses (ABR)) of two wild-type laboratory strains $(\mathrm{C} 57 \mathrm{BL} / 6 \mathrm{~N}$, postnatal day $(\mathrm{P}) 30$; and NMRI, P15-P20). We approximated their collective synaptic cochleograms by a quadratic function (Fig. 1b) to describe synapse number per IHC along the tonotopic axis (see Supplementary Table 1 online for fit coefficients). In gerbils (P28), we counted only synaptic ribbons because we were not able to stain postsynaptic glutamate receptor clusters reliably (Fig. 1c; Supplementary Fig. 1 and Supplementary Table 1). The synaptic cochleograms were then related to hearing thresholds as estimated by ABR recordings (Fig. 1b; mice were thereafter used for immunohistochemistry) or compound action potentials ${ }^{24}$ (Fig. 1c) using published place-frequency maps ${ }^{18,29}$. For both species, the synaptic cochleograms peaked within
Figure 2 Spatial distribution of afferent synapses within IHCs of one tonotopic region. (a) Cartoon illustrating the analysis and the use of cylindrical coordinates for descriptions of synapse position. (b) Four en face views of six midcochlear IHCs and their afferent synapses (red dots), overlaid after normalization of synapse coordinates in the $z$ axis (from the center of the nucleus to the basal end) and in the radius, $r$ (according to the width at nuclear level) of each IHC. Scale bars, $5 \mu \mathrm{m}$. (c) Histogram shows synapse number as a function of axial distance from the center of the cell's nucleus ( $z$ ) for a total of twenty-three midcochlear IHCs. (d) Polar scatter plot of 373 synapses mapped in cylindrical coordinates (radius $(r)$ and angle $(\theta)$ are displayed). (e) Polar histogram of synapse density in $36^{\circ}$ sectors. The sum of all 10 sectors equals the average synapse number of 16.2 per cell. (f) Histogram of the nearest neighbor distance distribution of 373 synapses.
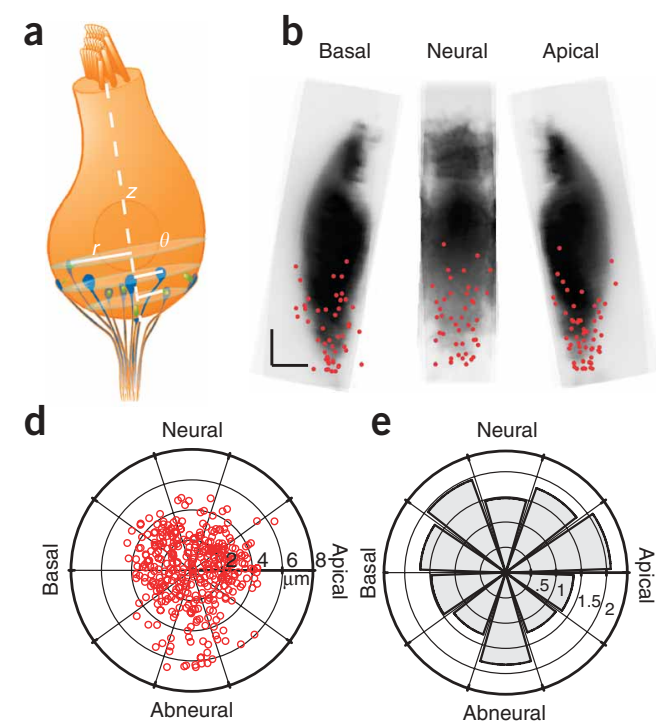

C
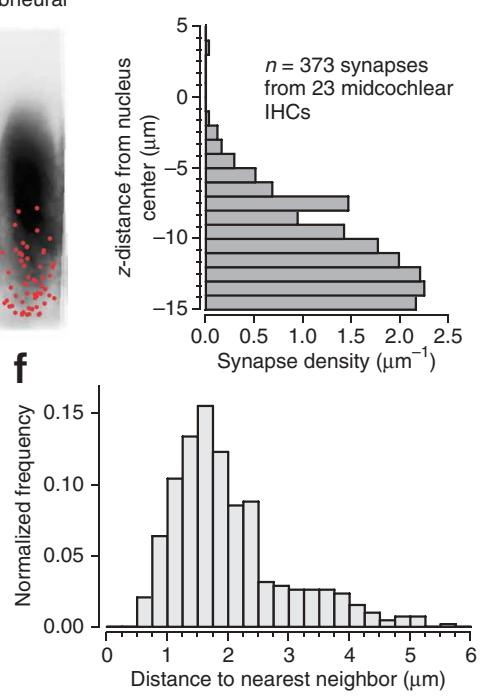

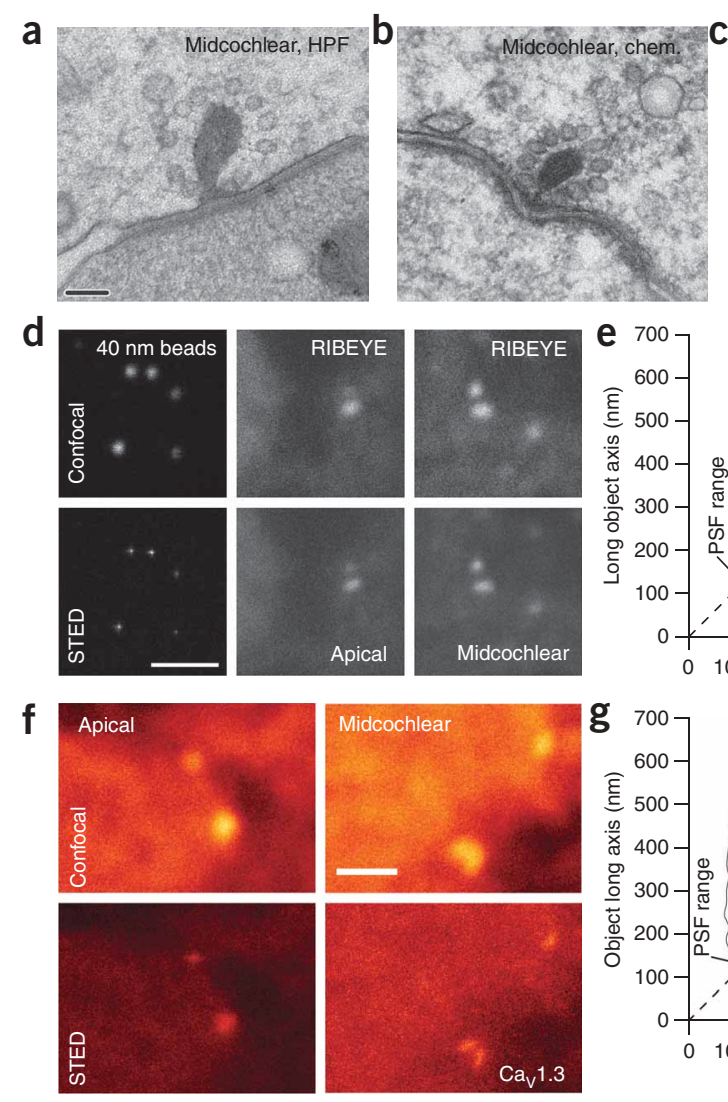

h Apical
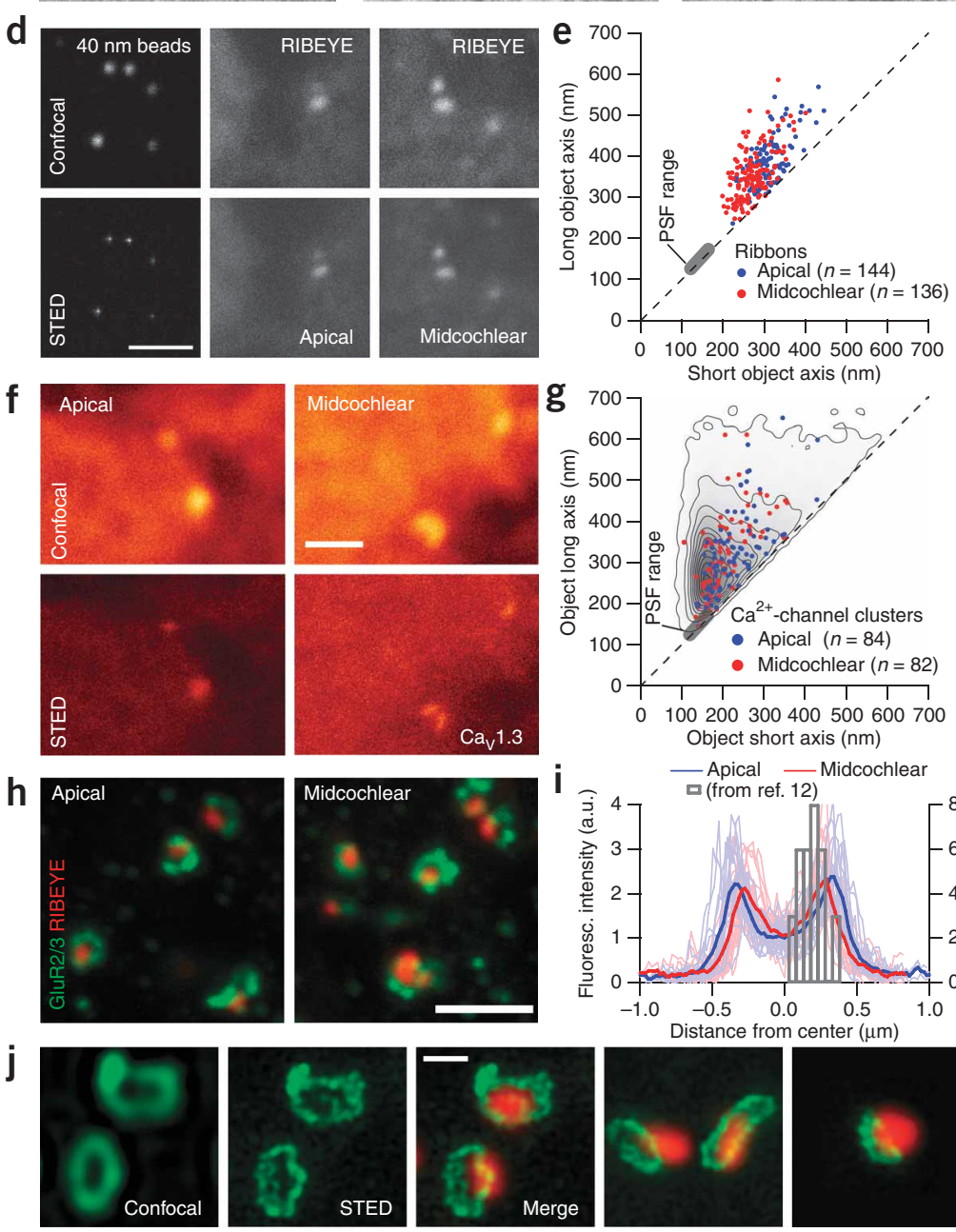

Object short axis (nm)
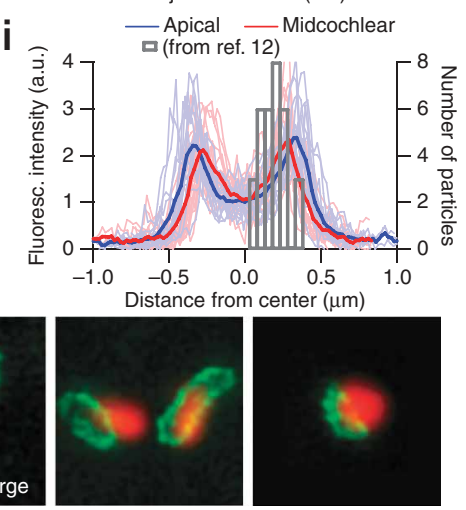

Figure 3 Ribbon synapse morphology in the apical cochlea $(100-400 \mu \mathrm{m})$ and mid-cochlea $(1,300-2,100 \mu \mathrm{m})$ of the mouse (P16-P21). (a-c) Representative electron micrographs of midcochlear (a,b) and apical (c) synapses, after either high-pressure rapid freeze and freezesubstitution (HPF, a) or aldehyde fixation (chem., b and c). Scale bar, $100 \mathrm{~nm}$. (d) Representative confocal (top) and STED (bottom) images of 40-nm beads (left) and fluorescently labeled synaptic ribbons of apical (middle) and midcochlear (right) IHCs. Scale bar, $2 \mu \mathrm{m}$. (e) Long versus short axes for apical (blue) and midcochlear (red) ribbons. Grey bar: resolution limit of the STED microscope in front (lower boundary) and behind (upper boundary) the sample. (f) Representative confocal (top) and STED (bottom) images of immunolabeled Cav1.3 clusters of an apical and a midcochlear IHC imaged 15-20 $\mu \mathrm{m}$ deep in the sample. Scale bar, $1 \mu \mathrm{m}$. The double cluster in the midcochlear IHC was associated with a very large RIBEYE signal (not shown). (g) Long versus short axes for apical (blue) and midcochlear Cav1.3 clusters (red). Expected density (Supplementary Fig. 5) overlaid as contour plot. (h-j) Imaging of postsynaptic AMPA receptor clusters (green, GluR2/3; red, RIBEYE) contacting apical and midcochlear IHCs. (h) Ring-like appearance of clusters oriented en face. Scale bar, $2 \mu \mathrm{m}$. (i) Fluorescence (fluoresc.) profiles from STED en face views of apical (blue) and midcochlear (red) clusters. Profiles were centered at half the distance of the two side-peaks for alignment. Gray bars, counts of immunogold particles as a function of distance from synapse center (rat IHC, taken from ref. 12); a.u., arbitrary units. (j) Two leftmost panels: representative deconvolved $x y$-confocal and STED sections of clusters. Middle panel: same cluster overlaid with confocal image of ribbon. Scale bar, $500 \mathrm{~nm}$. Two rightmost panels: more synapses.

along $\theta$. This is exemplified in views from all four sides of six overlaid midcochlear IHCs (Fig. 2b). We further described the synapse distribution as functions of $z$-position (Fig. 2c) and angle $\theta$ (Figs. 2d,e). The distribution of the three-dimensional nearest

the most sensitive region of the cochlea at $\sim 17$ (mice) or $\sim 24$ (gerbils) synapses per IHC and declined toward the cochlear apex and base.

Next, we studied the synapse distribution within apical (distance to cochlear apex $200 \pm 100 \mu \mathrm{m}, n=2$ mice; Supplementary Fig. 2 online) and midcochlear (1,700 $\pm 400 \mu \mathrm{m}, n=4$ mice; Fig. 2) IHCs. Stacks of confocal images obtained from organs of Corti immunolabeled for CtBP2/RIBEYE and calbindin-28k, a $\mathrm{Ca}^{2+}$-binding protein marking the IHC cytosol, were aligned according to the tonotopic axis (indicated by the row of IHCs) to identify synaptic ribbons and IHC nuclei (see Methods). Synapses were assigned to IHCs based on their distance to the center of the nearest IHC's nucleus. Data were discarded if assignments were ambiguous. The position of each synapse was expressed in cylindrical coordinates ( $z$-axial position, with $z=0$ at the center of the nucleus; radius $r$ and angle $\theta$ as illustrated in Fig. 2a) after normalizing the distance between the center of the nucleus and the basal end of each IHC to the respective mean value of all analyzed IHCs. We observed a strong base-to-apex decline in synapse abundance from the base of the IHC to its apex and a rather uniform distribution neighbor distance had a mean of $\sim 2 \mu \mathrm{m}$ and did not show obvious higher-order peaks (Fig. 2f, estimated before normalization of cell dimensions). The synapse distribution was similar for IHCs in the apex of the cochlea, except for a tendency of synapses to accumulate in the apical and basal IHC sectors (Supplementary Fig. 2).

\section{Synapse morphology as a function of tonotopic position}

The size of the synaptic ribbon largely determines the vesicle complement of each synapse (reviewed in ref. 3) and was previously used to approximate the area of the active zone over which $\mathrm{Ca}^{2+}$ channels are distributed $^{30,31}$. The size of postsynaptic AMPA receptor clusters is a key determinant of synaptic strength (for example, refs. 32,33). Thus, we explored IHC synapse morphology in apical and midcochlear regions using high-resolution fluorescence microscopy of immunolabeled IHC synapses, as well as electron microscopy.

Electron microscopy was performed on ultrathin sections of first apical turns of cochleae. First, we compared the synaptic ultrastructure after (i) chemical immersion fixation or (ii) high-pressure rapid freeze 
Table 1 Morphology of ribbons synapses in apical and midcochlear IHCs

\begin{tabular}{|c|c|c|c|}
\hline & Apical (mean \pm s.d.) & Midcochlear (mean \pm s.d.) & $P$-value \\
\hline Cav1.3 cluster long axis (STED) & $327 \pm 95 \mathrm{~nm}(n=84)$ & $320 \pm 97 \mathrm{~nm}(n=82)$ & $P=0.40$ \\
\hline Cav1.3 cluster short axis (STED) & $228 \pm 59 \mathrm{~nm}(n=84)$ & $208 \pm 55 \mathrm{~nm}(n=82)$ & $P<0.01$ \\
\hline Ribbon long axis (STED) & $379 \pm 62 \mathrm{~nm}(n=144)$ & $357 \pm 63 \mathrm{~nm}(n=136)$ & $P<0.001$ \\
\hline Ribbon long axis (EM) & $228 \pm 60 \mathrm{~nm}(n=29)$ & $223 \pm 59 \mathrm{~nm}(n=37)$ & $P=0.74$ \\
\hline Ribbon short axis (STED) & $299 \pm 46 \mathrm{~nm}(n=144)$ & $273 \pm 39 \mathrm{~nm}(n=136)$ & $P<0.001$ \\
\hline Ribbon short axis (EM) & $118 \pm 27 \mathrm{~nm}(n=29)$ & $117 \pm 36 \mathrm{~nm}(n=37)$ & $P=0.86$ \\
\hline Ribbon cross-section (EM) & $21,337 \pm 8,609 \mathrm{~nm}^{2}(n=29)$ & $20,495 \pm 9,795 \mathrm{~nm}^{2}(n=37)$ & $P=0.28$ \\
\hline Num $_{S V}<50 \mathrm{~nm}$ from ribbon (EM) & $15.1 \pm 3.5(n=27)$ & $12.7 \pm 3.7(n=34)$ & $P=0.01$ \\
\hline Num $_{S V}>50 \mathrm{~nm}$ from ribbon (EM) & $5.5 \pm 4.2(n=27)$ & $0.8 \pm 1.0(n=34)$ & $P<0.001$ \\
\hline SV diameter (EM, CF) & $41.4 \pm 5.7 \mathrm{~nm}(n=411 \mathrm{SVs})$ & $44.6 \pm 5.6 \mathrm{~nm}(n=431 \mathrm{SVs})$ & $P<0.001$ \\
\hline SV diameter (EM, HPF) & - & $48.7 \pm 8.2 \mathrm{~nm}(n=156 \mathrm{SVs})$ & \\
\hline GluR2/3 cluster (STED) 'size' & $884 \pm 15 \mathrm{~nm}(n=16)$ & $751 \pm 11 \mathrm{~nm}(n=19)$ & $P<0.01$ \\
\hline 'Width' & $180 \pm 0.71 \mathrm{~nm}$ & $193 \pm 0.89 \mathrm{~nm}$ & $P=0.39$ \\
\hline Ratio $_{\max / \min }$ & $2.5 \pm 0.9$ & $2.3 \pm 0.7$ & $P=0.45$ \\
\hline Postsynaptic density (EM) & $819 \pm 154 \mathrm{~nm}(n=29)$ & $888 \pm 200 \mathrm{~nm}(n=30)$ & $P=0.74$ \\
\hline
\end{tabular}

EM, electron microscopy; NumsV, number of synaptic vesicles; SV, synaptic vesicle; CF, chemical fixation; HPF, high pressure freeze; 'size' of GluR2/3 clusters, sum of peak-to-peak distance and average FWHM of the two peaks in a fluorescence profile of the cluster

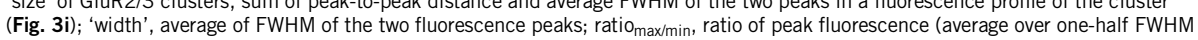
around the peak location) and minimal fluorescence (average over one-half FWHM around the minimum location).

and subsequent freeze substitution (Supplementary Fig. 3 and Supplementary Methods online) because the latter method may better preserve synapse morphology ${ }^{34}$. We did not observe obvious differences in the complement of vesicles; the mean vesicle diameter estimate in the chemically fixed tissue was smaller by less than 10\% (Fig. 3a,b; Table 1). Therefore, we used chemical fixation for further analyses. We did not detect significant differences in ribbon size and shape (short and long axes, cross-sectional area), nor in the length of the postsynaptic density (PSD) between midcochlear (Fig. 3a,b) and apical (Fig. 3c) IHC synapses (Table 1). We quantified the abundance of synaptic vesicles at the active zone for ribbon-associated $(<50 \mathrm{~nm}$ from ribbon) and unassociated ( $>50 \mathrm{~nm}$ from ribbon) vesicles. There were slightly more ribbon-associated vesicles and substantially more unassociated ones in apical synapses compared to midcochlear ones (Table 1).

Optical microscopy of immunolabeled subcellular structures enables high throughput analysis, providing a robust basis for statistical comparison. IHC ribbons, $\mathrm{Ca}^{2+}$ channel clusters and aspects of postsynaptic AMPA receptor clusters (see below) are at or below the resolution limit of confocal microscopy $(\sim 250 \mathrm{~nm})$. Therefore, we used high-resolution 4Pi microscopy (one-dimensional axial resolution $\sim 100 \mathrm{~nm}$; Supplementary Fig. 4 online) and STED microscopy (two-dimensional lateral resolution $\sim 50-150 \mathrm{~nm}$, Fig. 3; threedimensional spherical resolution $\sim 150 \mathrm{~nm}$, Supplementary Movie 1 online) in addition to confocal microscopy to compare those structures between apical and midcochlear synapses. Note that these measurements report apparent rather than absolute object size because of immunolabeling and fluorescence imaging, and thereby overestimate true object size. However, this does not impede the comparison between synapses of different tonotopic regions or sectors of IHCs.

To study ribbons, we analyzed 4Pi images stacks as described previously ${ }^{6}$. The analysis revealed overlapping distributions of apparent axial diameter of ribbons between apical and midcochlear IHCs, indicating similar ribbon size and shape (Supplementary Fig. 4). We fitted gaussian functions to the distributions and found that the means and s.d. values (for apical, $323 \pm 57 \mathrm{~nm}, n=193$ ribbons; for midcochlear, $324 \pm 62 \mathrm{~nm}, n=168$ ribbons) were indistinguishable. Compared to confocal microscopy implemented on the same microscope, STED yielded superior resolution (Fig. 3d,f; lateral dimensions of point spread function (PSF), $\sim 150 \times 150 \mathrm{~nm}$; axial dimension, $\sim 500 \mathrm{~nm}$ at the position of the synapses in the tissue for Fig. $\mathbf{3 d}-\mathbf{g}$ ). The resolution of the STED microscope decreases with the depth of penetration into the tissue and was controlled by measuring the PSF with $100-\mathrm{nm}$ fluorescent beads in front and behind the sample (Fig. 3e,g; Supplementary Fig. 4). The short and long axes of fluorescence spots in the STED sections were approximated as the full widths at half maximum (FWHM) of two orthogonal gaussian functions (Fig. 3e,g). They were slightly but significantly larger for apical ribbons (Table 1 and Supplementary Fig. 4). On average, the mean apparent axes ((short + long)/2) differed by $25 \mathrm{~nm}$ between the two locations. The ratios of long to short apparent axes were statistically indistinguishable (apical, $1.27 \pm 0.16$, $n=144$; midcochlear, $1.31 \pm 0.19, n=$ 136; $P=0.08)$ and were consistent with an ellipsoid ribbon structure. $\mathrm{Ca}_{\mathrm{V}} 1.3 \mathrm{Ca}^{2+}$ channels cluster at active zones of hair cells ${ }^{11,35}$. Using STED microscopy, we studied size and shape of immunolabeled $\mathrm{Ca}_{\mathrm{V}} 1.3$ clusters in IHCs at the two different tonotopic positions (Fig. 3f,g). The size of the observed fluorescent spots (two-dimensional FWHM) ranged between $140 \mathrm{~nm}$ and $650 \mathrm{~nm}$; hence, one of the object axes was typically above the resolution limit of this STED microscope but in many cases too small for estimation by confocal microscopy. Nearby $\mathrm{Ca}^{2+}$ channel clusters (Fig. 3f, right panel), which are readily resolved by STED microscopy but not discernable by confocal microscopy, existed rarely ( $<10 \%$ of all analyzed synapses). The average size and shape of the synaptic $\mathrm{Ca}_{V} 1.3$ clusters were similar for apical and midcochlear IHCs (Fig. 3g, Table 1 and Supplementary Fig. 5 online) (mean apparent axis: apical $278 \pm 71 \mathrm{~nm}, n=84$; midcochlear $264 \pm 69$ $\mathrm{nm}, n=82, P=0.10$; axis ratio: apical $1.45 \pm 0.30$; midcochlear $1.56 \pm$ $0.42 ; P=0.06)$. We modeled the two-dimensional projection of randomly oriented objects (after convolution with the point spread function of the STED microscope; Supplementary Fig. 5) because the real three-dimensional shape of the clusters cannot be readily deduced from the data. We simulated variably sized objects with several geometric shapes aiming to match the experimentally observed distributions of short and long axes (see Supplementary Fig. 5). The data could be reasonably well described by assuming a flat, oblate ellipsoid with diameter $420 \pm 130 \mathrm{~nm}$, short axis below the resolution limit (Fig. 3g). We cannot exclude the existence of subclusters in mice, as proposed for frog hair cells ${ }^{35}$, but, if present, those must be separated by less than $150 \mathrm{~nm}$.

AMPA receptor clusters, detected as GluR2/3 immunofluorescent spots, showed a ring-like shape when oriented in parallel with the $x y$ plane $($ Fig. $3 \mathbf{h}-\mathbf{j})$, indicating a gradient of receptor density in the plane of the PSD with an off-center maximum. The ring-like fluorescence pattern was confirmed by high-resolution three-dimensional STED microscopy (Supplementary Movie 1). We cannot entirely rule out lower accessibility to antibody labeling of AMPA receptors in the center of the synapse. However, we consider this highly unlikely, as a similar gradient of receptor density was found in a previous immunoelectron microscopy study of rat IHC afferent synapses ${ }^{12}$, where antigen accessibility should not be a concern owing to postembedding immunogold labeling of AMPA receptors in ultrathin sections. En face views of AMPA 

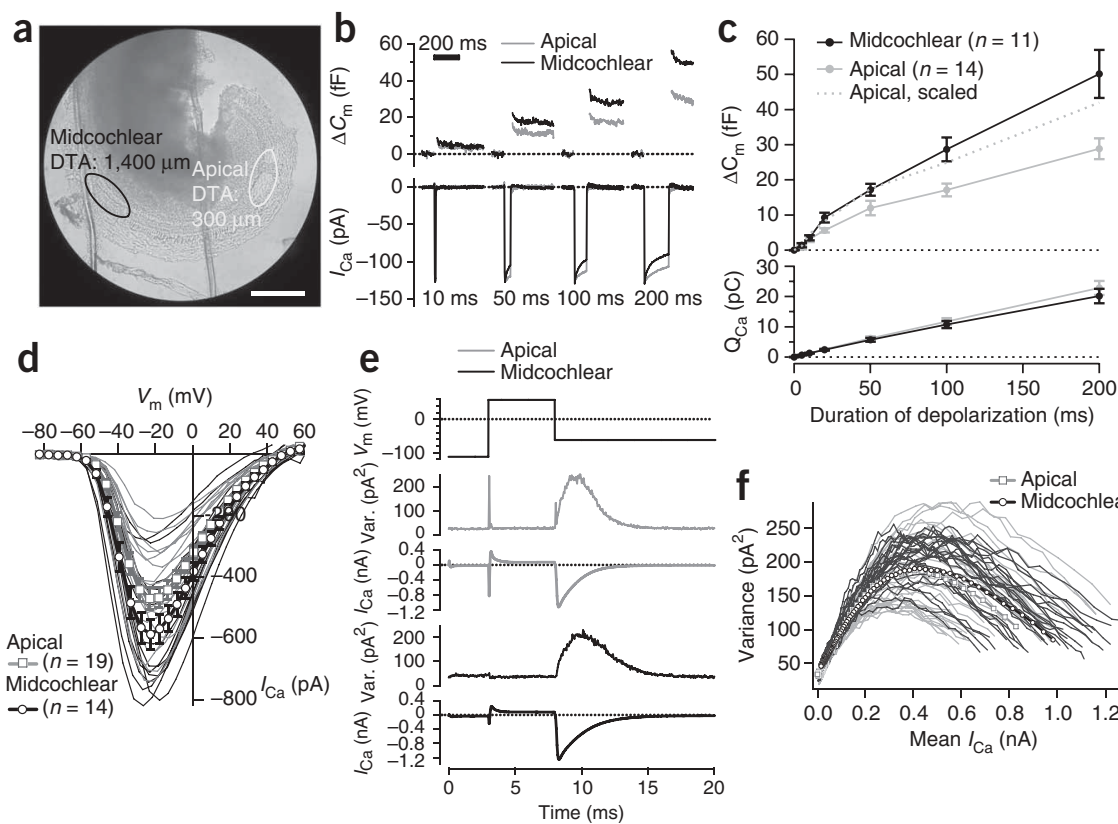

$\infty-(n=14)$

Figure 4 Exocytosis scales with the number of afferent synapses per IHC. (a) Distance-to-apex (DTA) measurement of representative tonotopic regions used for patch-clamp recordings, identified by locally removed rows of outer hair cells and pillar cells (ellipses). Scale bar, $200 \mu \mathrm{m}$. (b) Average membrane capacitance $\left(C_{\mathrm{m}}\right)$ traces (baseline subtracted) and calcium current $\left(I_{\mathrm{Ca}}\right)$ of apical $(n=11$; gray) and midcochlear ( $n=14$; black) IHCs in response to 10-, 50-, 100- and 200-ms depolarizations. (c) Mean exocytic $\Delta C_{m}$ (top) and $\mathrm{Ca}^{2+}$ current integrals (bottom) of the same cells as a function of stimulus duration. Scaled apical (dotted line) is $C_{\mathrm{m}}$ of apical IHCs multiplied by 1.44 (ratio of the number of synapses for midcochlear and apical IHCs). (d) Average current-voltage relationships (thick traces with symbols) of apical ( $n=19$, thin gray traces) and midcochlear ( $n=14$, thin black traces) IHCs in augmenting conditions (10 mM extracellular [Ca $\left.{ }^{2+}\right], 5 \mu \mathrm{M}$ BayK8644). (e,f) Analysis of $\mathrm{Ca}^{2+}$ tail current fluctuations in augmenting conditions: (e) Pulse protocol and typical examples of current ensemble variance and mean of an apical and a midcochlear IHC, respectively. (f) Variance-mean relationships of all apical and midcochlear IHCs (light and dark thin traces, respectively) and grand mean for both cell populations (thick traces with symbols). Error bars, s.e.m.

receptor clusters were analyzed by fitting the STED (Fig. 3i) fluorescence profiles with a sum of two gaussians. As expected, the limited resolution of confocal microscopy led to an overestimation of the peak width (confocal $\sim 350 \mathrm{~nm}$; STED $\sim 180 \mathrm{~nm}$ (lateral resolution $<80 \mathrm{~nm}$ for this microscope)), and thus we based further analysis solely on STED microscopy. Whereas the width of the peaks was statistically indistinguishable between apical and midcochlear clusters, we found slightly larger peak-to-peak distances (apical $683 \pm 84 \mathrm{~nm}, n=16$; midcochlear $562 \pm 85 \mathrm{~nm}, n=19$ ) and total sizes for clusters of apical synapses (Table 1). The outer diameter of the cluster roughly matched the length of the PSD as measured by electron microscopy (Table 1), indicating that AMPA receptors populate most of the PSD. On average, the fluorescence peaks were about twofold brighter than the center, for both apical and midcochlear AMPA receptor clusters (Table 1).

\section{Tonotopy of IHC presynaptic physiology}

Recent studies have used patch-clamp recordings of $\mathrm{Ca}^{2+}$ currents and membrane capacitance changes $\left(\Delta C_{\mathrm{m}}\right)$ to study the presynaptic properties of hair cells of various species (for review, see ref. 3 ). It remains to be clarified whether this technique reports exclusively synaptic $\mathrm{Ca}^{2+}$ current and exocytosis in IHCs ${ }^{35-37}$ or also a significant extrasynaptic fraction of channels and/or vesicles $5,6,11,38,39$. We also wondered whether, on top of the number of synapses, their function may also vary along the cochlea's tonotopic axis ${ }^{15,16,30,31,37}$. We first studied whole-cell $\mathrm{Ca}^{2+}$ currents and exocytic membrane capacitance changes
$\left(\Delta C_{\mathrm{m}}\right)$ in IHCs from those apical and midcochlear regions (Fig. 4a) that had also been morphologically investigated (Figs. 1-3). IHCs at these two locations had $14.0 \pm 0.3$ (midcochlear) and $9.7 \pm 0.7$ (apical) synapses, respectively, and, as judged from their resting membrane capacitance (midcochlear IHCs $8.7 \pm 1.1 \mathrm{pF}, n=11$; versus apical IHCs 7.7 $\pm 0.7 \mathrm{pF}, n=14$ ), they differed slightly in size. We recorded their $\mathrm{Ca}^{2+}$ currents and membrane capacitance increments in the perforated-patch configuration in response to step depolarizations to $-14 \mathrm{mV}$ (Fig. 4b,c). For most stimuli, we identified larger exocytic $\Delta C_{\mathrm{m}}$ for midcochlear IHCs. Exocytosis of midcochlear and apical IHCs could be roughly matched when scaling the responses of apical IHCs by the ratio of the number of synapses (1.44; Fig. 4c).

Midcochlear IHCs tended to show larger peak $\mathrm{Ca}^{2+}$ currents (ratio of peak currents; under non-augmenting conditions $(2 \mathrm{mM}$ $\left[\mathrm{Ca}^{2+}\right]$, perforated-patch): 1.1, augmenting conditions $\left(10 \mathrm{mM}\right.$ extracellular $\left[\mathrm{Ca}^{2+}\right]$, $5 \mu \mathrm{M}$ BayK8644, whole-cell): 1.2, Fig. 4d and Supplementary Table 2 online). The difference in $\mathrm{Ca}^{2+}$ current integrals (Fig. 4c) did not reach statistical significance, probably because of the more pronounced $\mathrm{Ca}^{2+}$ current inactivation in midcochlear IHCs (Fig. 4b). Neither were voltage dependence and kinetics of $\mathrm{Ca}^{2+}$ current activation different (Supplementary Fig. 6 online). Assuming an exclusively synaptic localization of $\mathrm{Ca}^{2+}$ channels and given the roughly comparable size of synaptic clusters of $\mathrm{Ca}^{2+}$ channels (Fig. 3), one would have expected the peak $\mathrm{Ca}^{2+}$ currents in midcochlear and apical IHCs to scale with the number of synapses, as seen for exocytosis. The observed discrepancy could be due to differences in channel open probability or single-channel current among IHCs at the two positions. When analyzing fluctuations among repetitively evoked $\mathrm{Ca}^{2+}$ tail currents under augmenting conditions (Fig. 4e,f), we found more $\mathrm{Ca}^{2+}$ channels (1.34:1, midcochlear/apical), a slightly higher open probability and a somewhat smaller single-channel current in the midcochlear cells (see Supplementary Table 2).

Conclusions from whole-cell recordings on synaptic $\mathrm{Ca}^{2+}$ channels would be confounded by the presence of extrasynaptic $\mathrm{Ca}^{2+}$ channels. To directly compare synaptic $\mathrm{Ca}^{2+}$ signaling at different tonotopic places, we used fast confocal imaging of IHCs loaded with Fluo-5N. Depolarization caused the rapid appearance of spatially restricted fluorescence hotspots in the basolateral compartment of IHCs from hearing mice (Fig. 5a) that were mediated by voltage-gated $\mathrm{Ca}^{2+}$ influx and localized with ribbons ${ }^{40}$, as previously described in nonmammalian hair cells ${ }^{36,41,42}$. Once such $\mathrm{Ca}^{2+}$ microdomains were identified in confocal sections, we used spot detection (continuous read-out of fluorescence from the maximum-intensity location inside the $\mathrm{Ca}^{2+}$ microdomain (Fig. 5b,c) and perpendicular line scans (Fig. 5d,e) to study the kinetics (Fig. 5b), voltage dependence (Fig. 5c) and FWHM (Fig. 5e) of these synaptic $\mathrm{Ca}^{2+}$ signals during 20-ms depolarizations). On average, these properties were indistinguishable between apical and midcochlear IHCs (Table 2). 

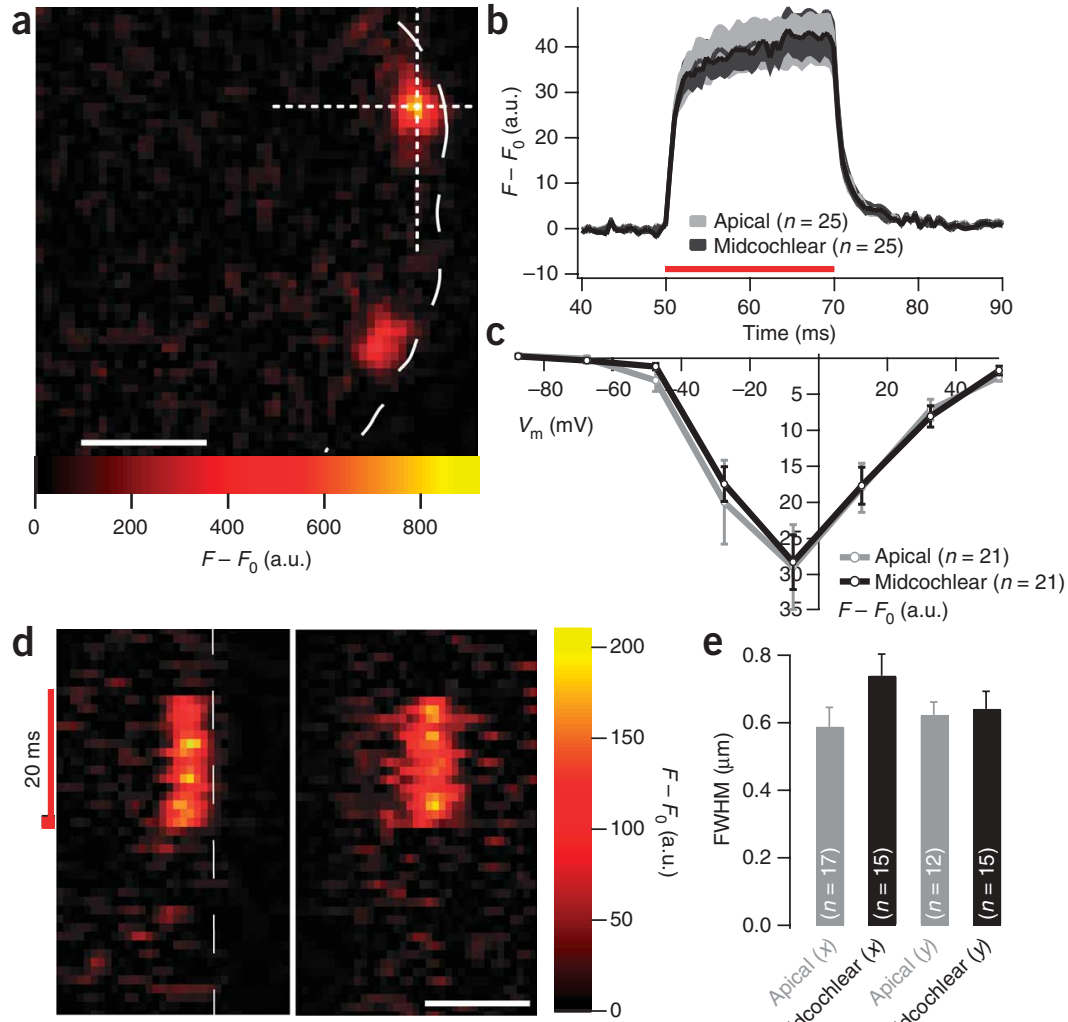

e

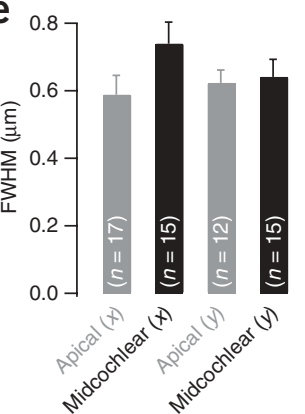

Figure 5 Synaptic $\mathrm{Ca}^{2+}$ signals are comparable at different tonotopic locations. (a) $x y$ scan reveals hotspots of Fluo- $5 \mathrm{~N}$ fluorescence in the base of a P14 IHC (loaded with $400 \mu \mathrm{M}$ Fluo- $5 \mathrm{~N}$ and $2 \mathrm{mM}$ EGTA; dotted line, IHC border) during membrane depolarization to $-7 \mathrm{mV}$ (red bar, time of depolarization). Resting fluorescence $\left(F_{0}\right)$ was subtracted. Scale bar, $2 \mu \mathrm{m}$; a.u., arbitrary fluorescence intensity units. (b) Fluorescence time courses for $\mathrm{Ca}^{2+}$ microdomains in apical (gray) and midcochlear (black) IHCs. Data were obtained at high temporal resolution at the brightest pixel of the hotspot (spot detection; see Methods) (dotted crosshairs in a), on which the laser beam was parked. Red bar, depolarization to $-7 \mathrm{mV}$. (c) $\mathrm{Ca}^{2+}$ microdomain amplitude as a function of membrane potential. The average $\Delta F$ amplitude over the last $15 \mathrm{~ms}$ of the respective spot detection response to $20 \mathrm{~ms}$ depolarizations is plotted. (d) Line scan. Dashed line in left panel, $\mathrm{IHC}$ border. (e) Spatial extent of $\mathrm{Ca}^{2+}$ microdomains. FWHM values were obtained by fits of gaussian functions to time-averaged (20 ms) line scans (d). Error bars, s.e.m.

and starting $x y$-position in repetitive $x y$-scans, followed by (ii) selection of the brightest pixel identified during lateral spot displacement along one dimension (from the starting position). Moreover, we obtained a second estimate by fitting gaussian functions to the line scans, and we found the variability comparable (Supplementary Table 3). The scatter of fluorescence amplitude (see Methods) for 65 individual synapses in 20 apical and midcochlear IHCs responding to $20 \mathrm{~ms}$ depolarizations to $-7 \mathrm{mV}$ (Fig. 6a) had a coefficient of variation $(\mathrm{CV})$ of 0.63 . Similar variability was also found for the background-normalized data $\left(\Delta F / F_{0}, \mathrm{CV}=0.61\right)$, arguing against a contribution of variation in dye concentration.

Even synapses of an individual IHC differed up to tenfold in their fluorescence amplitude (Fig. 6b; maximum/minimum $\Delta F$ on average, 4.5 -fold; average $\mathrm{CV}=0.57$ for seven apical IHCs). The variance among the fluorescence changes was not systematic with time (Fig. 6b), arguing against $\mathrm{Ca}^{2+}$ current run-down, indicator bleaching, or buffer saturation being sizeable contributors. The trial-to-trial variability of $\Delta F$ of the same $\mathrm{Ca}^{2+}$ microdomain

Peak $\Delta F\left(F-F_{0}\right.$, a.u. $)$

$A_{\text {onset,fast }}$ (ms)

$A_{\text {onset,slow (ms) }}$

$A_{\text {onset,fast }} / A_{\text {onset,slow }}$

$A_{\text {decay,fast }}(\mathrm{ms})$

$A_{\text {decay,slow }}(\mathrm{ms})$

$A_{\text {decay,fast }} / A_{\text {decay,slow }}$

$$
\begin{aligned}
39.1 & \pm 5.3(n=25) \\
1.0 & \pm 0.1(n=25 / 25) \\
17 & \pm 8(n=8 / 25) \\
2.3 & \pm 0.7(n=8 / 25) \\
1.1 & \pm 0.1(n=25 / 25) \\
8 & \pm 1(n=13 / 25) \\
5.1 & \pm 0.8(n=13 / 25)
\end{aligned}
$$

Midcochlear (mean \pm s.e.m.)

$P$-value

$V_{1 / 2}(\mathrm{mV})$

$-26.1 \pm 3.8(n=13)$

$7.3 \pm 1.4(n=13)$

$$
\begin{aligned}
39.3 & \pm 4.5(n=25) \\
1.0 & \pm 0.1(n=25 / 25) \\
60 & \pm 38(n=12 / 25) \\
1.5 & \pm 0.4(n=12 / 25) \\
1.0 & \pm 0.1(n=25 / 25) \\
28 & \pm 10(n=13 / 25) \\
3.9 & \pm 0.7(n=13 / 25) \\
-26.1 & \pm 2.0(n=13) \\
6.3 & \pm 0.6(n=13)
\end{aligned}
$$

$P=0.97$

$P=0.16$

$P=0.16$ was small $(\mathrm{CV}=0.09)$. We found only weak, although significant, correlations

0.16 between fast-onset kinetics and fluores-

$P=0.24$ cence amplitude (Fig. 6c; linear correlation

$P=0.11$ coefficient $\left.P_{r}=0.28, P<0.05\right)$, and

$P=0.27 \quad$ FWHM of $\mathrm{Ca}^{2+}$ microdomains and their

$P=0.28$

$P=0.65$

Line scan

$x$ FWHM $(\mu \mathrm{m})$

$0.6 \pm 0.1(n=17)$

$0.7 \pm 0.1(n=15)$

$P=0.09$ $y$ FWHM ( $\mu \mathrm{m})$

$0.6 \pm 0.1(n=12)$

$P=0.35$

Exponential fitting was used to estimate the on- and off-kinetics of the synaptic $\mathrm{Ca}^{2+}$ microdomain signals. A double-exponential fit was accepted if the time constants differed by at least a factor of 3 and if both components were sizeable. $A$, amplitude of the fluorescence component indicated in the subscript; $V_{1 / 2}$, voltage of half-maximal fluorescence increase; slope, steepness of the voltage-dependent increase of the fluorescence change. $V_{1 / 2}$ and slope were obtained by fitting Boltzmann functions to the fluorescence-voltage relationships (Fig. 5c). FWHM, full width at half-maximum of the time-averaged $\mathrm{Ca}^{2+}$ microdomain fluorescence. $x$ and $y$ designate the directions of the orthogonal line scans; a.u., arbitrary fluorescence intensity units. fluorescence amplitude (Fig. 6d; $P_{r}=$ $-0.23, P<0.05)$. Taken together, these correlations argue against a substantial contribution of defocus from the $\mathrm{Ca}^{2+}$ microdomain's center to the observed variance. We therefore interpret these differences to mostly reflect genuine variance among $\mathrm{Ca}^{2+}$ signals of the individual synapses.

Next, we asked whether active zones with different properties segregated along the perimeter of the IHC, as previously 
a
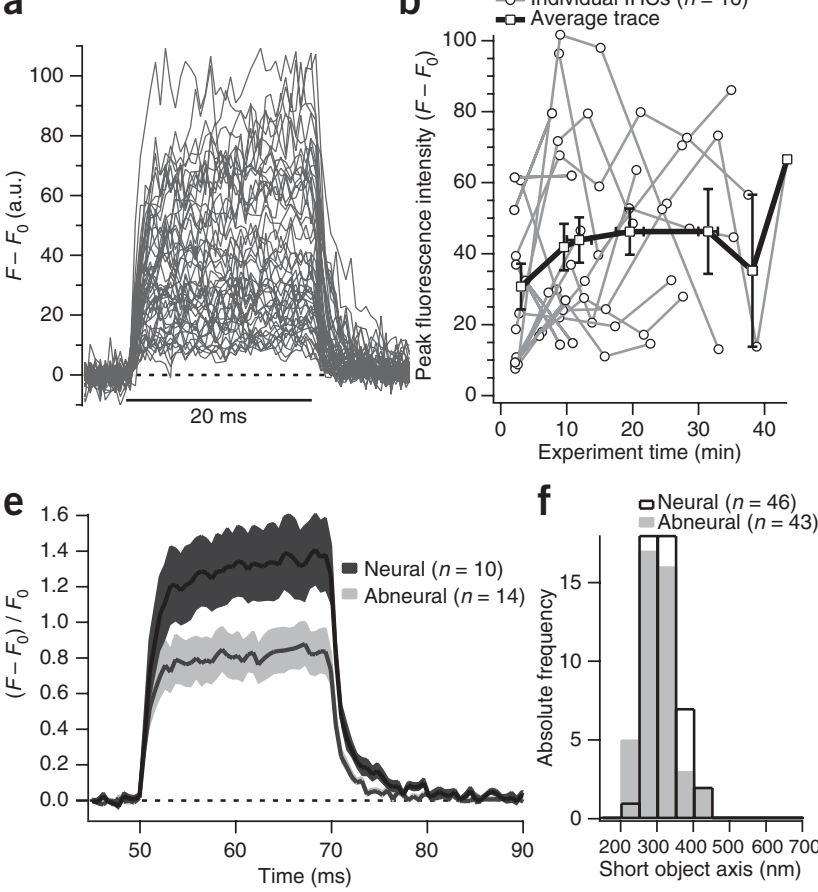

suggested in the $\mathrm{cat}^{24}$. We used confocal $\mathrm{Ca}^{2+}$ imaging and STED microscopy of immunolabeled ribbons in the neural and abneural $60^{\circ}$ sectors to test for potential functional and morphological differences of the respective synapses. Abneural synapses on average showed a smaller fluorescence increment (Fig. 6e; $\Delta F / F_{0}$ neural/abneural ratio, 1.6). The distributions of short (Fig. 6f) and long axes of ribbons (Fig. 6g) were indistinguishable, suggesting that the average ribbon size did not differ among neural and abneural synapses in mouse IHCs.

\section{DISCUSSION}

Our findings demonstrated that tonotopic variations in the sensitivity of the mammalian cochlea are paralleled by the density of its afferent innervation. We present nanoscale estimates of the size of ribbons, $\mathrm{Ca}^{2+}$ channel and AMPA receptor clusters at the IHC synapse for two different tonotopic regions. Although substantially changing in number, IHC synapses in these cochlear regions differ only slightly in structure. Exocytosis of IHCs scaled with the number of synapses, which, together with the comparable average properties of presynaptic $\mathrm{Ca}^{2+}$ signals, suggests a fairly uniform average synapse function in these two regions and also, presumably, further along the cochlea's tonotopic axis. However, $\mathrm{Ca}^{2+}$ signals showed substantial heterogeneity among synapses of an individual IHC, providing a potential presynaptic substrate for the divergent spiking properties of the SGNs that are driven by a given IHC.

Quantitative light microscopy of whole-mounted organs of Corti allowed assembly of continuous synaptic cochleograms for mouse (high-frequency hearing) and gerbil (low-frequency hearing) with a sample size and frequency resolution exceeding those of previous electron microscopy studies ${ }^{17,20,21,43}$. Synaptic cochleograms were well described by quadratic functions, providing a simple and useful tool for future studies on cochlear neurotransmission. The shape of the mouse synaptic cochleogram roughly followed that of the mouse behavioral audiogram ${ }^{44}$, that of the neural population audiogram (for example, Fig. 1) and that of the distortion product otoacoustic emission audiogram (for example, ref. 45). We interpret these findings as better neural sampling from the most sensitive cochlear regions. In
C

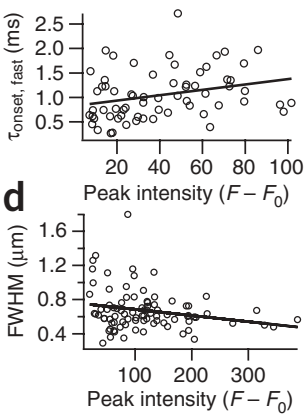

g

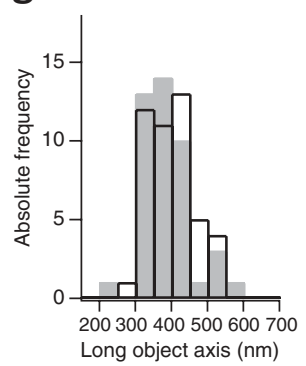

Figure 6 Intracellular variability of synaptic $\mathrm{Ca}$ microdomains. (a) Time course of fluorescence at the center of $\mathrm{Ca}^{2+}$ microdomains during 20-ms depolarization to peak $\mathrm{Ca}^{2+}$ current potential ( $-7 \mathrm{mV}$, as described in Fig. $5 \mathrm{c} ; n=65 \mathrm{Ca}^{2+}$ microdomains in $20 \mathrm{IHCs}$ ); a.u., arbitrary fluorescence intensity units. (b) $\mathrm{Ca}^{2+}$ microdomain amplitude within individual IHCs as a function of time of acquisition. (c,d) $\mathrm{Ca}^{2+}$ microdomain amplitude ( $\Delta F$ amplitude over the last $15 \mathrm{~ms}$ of data in a) correlates weakly with fast onset kinetics (c, linear correlation coefficient $\left.P_{r}=0.28, n=65\right)$ and with its FWHM (d; $\left.P_{r}=0.23, n=87\right) . \Delta F$ amplitude and FWHM were obtained by fitting gaussian functions to time-averaged line scans. (e) Differences in average peak $\mathrm{Ca}^{2+}$ microdomain amplitude between neural and abneural synapses $(P<0.05$, for peak amplitude). Gray bands, s.e.m. $(\mathbf{f}, \mathbf{g})$ Overlapping distributions of short and long ribbon axes of neural and abneural synapses of apical IHCs (distance to apex, 100-300 $\mu \mathrm{m}$ ) as estimated by STED microscopy.

the mouse, this region encodes the range of $\sim 10-20 \mathrm{kHz}$, which is important, for example, for the perception of wriggling calls of mouse pups ${ }^{46}$. Each SGN conveys information transmitted by one hair cell synapse to several neurons of the cochlear nucleus, which in turn integrate information from several SGNs. The reliability and acuity of afferent information provided by a region of the cochlea will increase with the number of innervating SGNs.

Here we took advantage of the tonotopic gradient of synapse number but uniform average presynaptic morphology in the mouse cochlea to ask whether whole-cell $\mathrm{Ca}^{2+}$ current and exocytic $\Delta C_{\mathrm{m}}$ scaled with the number of ribbon synapses, as would be expected if both occurred exclusively at the synapse. This was indeed found to be the case for exocytosis, as previously described for turtle hair cells ${ }^{37}$. Although we cannot exclude some contribution of vesicles fusing outside the active zone to exocytosis seen during prolonged stimulation ${ }^{3,47}$, we suppose that much of this sustained release occurs at the synapse. This view is supported by paired pre- and postsynaptic recordings from rat IHC synapses ${ }^{8}$. These data revealed AMPA receptor current integrals that were compatible with the notion of sustained exocytosis-as reported by capacitance measurements-reflecting synaptic release.

Our STED data suggest that, at the IHC synapse, AMPA receptors have a peripheral, 'ring-like' density maximum, which correlates with electron microscopy data on the rat cochlea ${ }^{12}$. The observed receptor distribution seems well suited for the efficient detection of glutamate release, in particular if this release occurs preferentially at the circumference of the ribbon ${ }^{35}$. It deviates from the uniform receptor distribution of glutamatergic CNS synapses suggested by immunoelectron microscopy (ref. 12; see review in ref. 48).

The $\mathrm{Ca}^{2+}$ current integral was not significantly different between apical and midcochlear IHCs. In part, this can be attributed to the stronger $\mathrm{Ca}^{2+}$ current inactivation in IHCs in the higher frequency region, which is consistent with findings in the gerbil ${ }^{49}$. Still, even the initial $\mathrm{Ca}^{2+}$ current was somewhat less correlated to synapse number than exocytosis. Similar findings were obtained in the turtle cochlea, where low-frequency synapses were assumed to mediate larger $\mathrm{Ca}^{2+}$ influx ${ }^{37}$. Unlike that study, we conclude, based on analysis of $\mathrm{Ca}^{2+}$ current, synaptic $\mathrm{Ca}^{2+}$ signals and $\mathrm{Ca}^{2+}$ channel cluster size that, except for inactivation, $\mathrm{Ca}^{2+}$ signaling of the average mouse IHC synapse is structurally and functionally similar 
in the two frequency regions. The existence of extrasynaptic $\mathrm{Ca}^{2+}$ channels, as suggested by single channel recordings from bullfrog hair cells ${ }^{39}$, is likely to explain the small remaining mismatch between the scaling of number of $\mathrm{Ca}^{2+}$ channels (1.34) and number of synapses (1.44).

Using various approaches, we compared, at two different frequency regions, key morphological determinants of IHC synaptic transmission, including synaptic ribbons and vesicle complement, synaptic $\mathrm{Ca}^{2+}$ channel clusters and postsynaptic density. We found only subtle differences (Fig. 3, Table 1), all indicative of slightly larger IHC synapses in the apex of the cochlea. Testing the functional relevance of these tonotopic differences requires more sensitive detection, such as in paired pre- and postsynaptic recordings ${ }^{8}$.

However, we found considerable variability for all of the investigated morphological and functional synapse parameters (Fig. 3, Tables 1 and 2, Supplementary Table 3 and Supplementary Fig. 4 and 5). Although this limits detection of small systematic changes along the tonotopic axis, it probably relates to the important question in auditory research of the mechanisms behind the different functional properties of SGNs. Pioneering work primarily in the cat cochlea has identified morphological correlates for high and low spontaneous firing rate SGNs (for example, active zone morphology, abneural versus neural insertion; diameter and mitochondrial content of the peripheral axon) ${ }^{14,22,24,43}$. In the present study, we observed slightly lower synaptic $\mathrm{Ca}^{2+}$ signals in the abneural sector of IHCs and found ribbon sizes to be comparable for neural and abneural synapses. These findings seem hard to reconcile with a systematically higher activity of abneural synapses in mouse IHCs, for which one might have expected larger ribbons providing more releasable vesicles ${ }^{3}$ and more $\mathrm{Ca}^{2+}$ channels.

Nevertheless, our experiments revealed substantial heterogeneity between $\mathrm{Ca}^{2+}$ signals at active zones within individual IHCs (Fig. 6b and Supplementary Table 3 ). Furthermore, we found a large variation of $\mathrm{Ca}_{\mathrm{V}} 1.3$ channel cluster size $(\mathrm{CV}=0.3)$, and, assuming a constant channel density in the cluster, we propose that differences in channel number contribute to this heterogeneity. Placement of synapses with different properties seems rather random in mouse IHC. Future studies combining imaging of synaptic $\mathrm{Ca}^{2+}$ signals with readouts of transmitter release and/or postsynaptic response ${ }^{7,8}$ will test how this translates into differences of transmitter release among the synapses of an individual mouse IHC.

\section{METHODS}

Animals. C57BL/6 and NMRI (Naval Medical Research Institute) mice aged 2-4 weeks and 4-week-old gerbils were used for experiments. Animal experiments complied with national animal care guidelines and were approved by the University of Göttingen Board for animal welfare and the animal welfare office of the state of Lower Saxony.

\section{Auditory brainstem responses. See Supplementary Methods.}

Patch-clamp and confocal $\mathrm{Ca}^{2+}$ imaging. IHCs from apical coils of freshly dissected organs of Corti from NMRI and C57BL/6 mice (P14-18) were patchclamped as described ${ }^{5}$. The pipette solution for perforated-patch recordings contained (in mM) 140 cesium gluconate, 13 tetraethylammonium (TEA)-Cl, $10 \mathrm{CsOH}$-HEPES buffer, $1 \mathrm{MgCl}_{2}$, and $250 \mu \mathrm{g} / \mathrm{ml}$ amphotericin $\mathrm{B}, \mathrm{pH}$ 7.2. The pipette solution for whole cell recordings contained (in $\mathrm{mM}$ ) 135 cesium glutamate, 13 TEA-Cl, 20 CsOH-HEPES, $1 \mathrm{MgCl}_{2}$, 2 Mg-ATP, 0.3 Na-GTP, 2 EGTA (10 for biophysical analysis of $\mathrm{Ca}^{2+}$ currents), 0.4 Fluo-5N (penta- $\mathrm{K}^{+}$ salt, Invitrogen; for confocal imaging), $\mathrm{pH}$ 7.0. The extracellular solution contained (in mM) $105 \mathrm{NaCl}, 35 \mathrm{TEA}-\mathrm{Cl}, 2.8 \mathrm{KCl}, 2 \mathrm{CaCl}_{2}$ (10 for fluctuation analysis, 5 for confocal imaging, balanced by $\mathrm{NaCl}$ ), $1 \mathrm{MgCl}_{2}, 10 \mathrm{NaOH}-$ HEPES, 10 D-glucose and 0.005 BayK8644 (Tocris, for fluctuation analysis), pH 7.2 (7.3 for whole-cell recordings). EPC-9 amplifiers controlled by Pulse or
Patchmaster software (HEKA Elektronik) were used for measurements. All voltages were corrected for liquid-junction potentials. Currents were low-pass filtered at $14 \mathrm{kHz}$ and $2 \mathrm{kHz}$ and sampled at $100 \mathrm{kHz}$ and $10 \mathrm{kHz}$ for whole-cell recordings and perforated-patch recordings, respectively. Cells that showed a holding current exceeding $-50 \mathrm{pA}$ were discarded from analysis. $\mathrm{Ca}^{2+}$ currents were further isolated using a $\mathrm{P} / \mathrm{n}$ protocol. Series resistance was required to be below $30 \mathrm{M} \Omega$ for perforated-patch experiments and averaged 10.0 $\pm 0.5 \mathrm{M} \Omega$ $(n=59)$ in the fluctuation analysis experiments. Fluctuation analysis was performed similarly to that previously described ${ }^{11}$. To account for channel gating-related and filter-induced correlations between neighboring currentvariance data points, we fitted the variance-over-mean data by an estimated generalized least-squares method (Supplementary Table 2). The first $600 \mu$ s of the tail current routinely were discarded. To avoid errors introduced by remaining, uncancelled capacitive transients, we subtracted a scaled version of the transient that was evident at the beginning of the depolarization step (see Fig. 4e) from the mean current trace (after calculation of the ensemble variance). Membrane capacitance increments $\left(\Delta C_{\mathrm{m}}\right)$ were measured as previously described ${ }^{5}$. Interstimulus periods were $2-3 \mathrm{~s}$ between sweeps, 1-2 min between ensembles for confocal imaging, and 30-70 s for exocytosis measurements.

Confocal $\mathrm{Ca}^{2+}$ imaging was performed as described ${ }^{40}$, using a Fluoview 300 confocal scanner mounted on an upright microscope (BX50WI) equipped with a 0.9 numerical aperture (NA), $\times 60$, water immersion objective (all Olympus) and a 50-mW, 488-nm, solid-state laser (Cyan, Newport-Spectraphysics). Fluorescent hotspots were identified in $x y$-scans during 200-ms depolarizations $(0.5 \%$ of maximum laser intensity) and further characterized using spot detection ('point scan' mode of the confocal scanner, temporally averaged to yield an effective sampling rate of $1.85 \mathrm{kHz}, 0.05 \%$ of maximum laser intensity) and line scans (at a rate of $0.74 \mathrm{kHz}, 0.25 \%$ of maximum laser intensity). Spot detection measurements and line scans were repeated 5 and 10 times, respectively, to improve signal-to-noise ratio. Peak $\Delta F$ estimates of spot detection measurements were obtained after repetitive boxcar smoothing (2-ms box). On average, we characterized 3.1 and 1.7 spots per IHC for apical-basal and neural-abneural comparisons, respectively. The average $\mathrm{Ca}^{2+}$ current rundown at the end of the experiment was $30 \%$ of the maximum current. For investigating intracellular differences, $\mathrm{Ca}^{2+}$ microdomain characterization was followed by acquisition of a $z$-stack of the indicator-filled cell so that its location could be retrieved.

Immunohistochemistry. Immunostaining was performed as previously described $^{6}$. Briefly, the freshly dissected apical cochlear turns were fixed with $4 \%$ paraformaldehyde for $1 \mathrm{~h}$ on ice, with $2 \%$ formaldehyde for $10 \mathrm{~min}$ at $20-22{ }^{\circ} \mathrm{C}$ (for staining with antibody to GluR2/3 (anti-GluR2/3)), or for $25 \mathrm{~min}$ in $99 \%$ methanol at $-20{ }^{\circ} \mathrm{C}$ (for anti-Ca 1.3 ). For harvesting the fulllength organs of Corti, cochleae were fixed by cochlear perfusion with $2 \%$ formaldehyde for $10 \mathrm{~min}$. The following antibodies were used: mouse IgG1 anti-CtBP2 (also recognizing the ribbon protein RIBEYE; BD Biosciences, 1:150), rabbit anti-GluR2/3 (Chemicon, 1:200), rabbit anti-calbindin (Swant, 1:400), rabbit anti-Cav1.3 (Alomone Labs, 1:75) and secondary Alexa Fluor 488- and Alexa Fluor 568-labeled antibodies (Molecular Probes, 1:200) as well as Atto-647N (AttoTech, 1:60 dilution in PBS with addition of $2 \%$ normal goat serum) for STED microscopy. In some experiments, nuclei were stained with Hoechst 34580 (Molecular Probes, 1:1,000).

Confocal, 4Pi and STED microscopy. Confocal morphological images were acquired using a laser scanning confocal microscope (Leica TCS SP5, Leica Microsystems) with 405-nm (diode), 488-nm (argon) and 561-nm (diodepumped solid state) lasers for excitation and a $\times 63$ oil immersion objective $(\mathrm{NA}=1.4)$. Whole-mount preparations of the organ of Corti allowed us to analyze several IHCs in a row, as previously described ${ }^{6}$. For three-dimensional reconstructions of the specimen, $z$-axis stacks of two-dimensional images were taken with a step size of $0.049 \mu \mathrm{m}, 0.2 \mu \mathrm{m}$ or $0.3 \mu \mathrm{m}$. Multifocal $4 \mathrm{Pi}$ microscopy with water immersion lenses (NA 1.2) at a two-photon excitation wavelength of $870 \mathrm{~nm}$ (average power, $1.5 \mathrm{~mW}$ for each of the $4 \mathrm{Pi}$ foci) was performed at a custom microscope as described ${ }^{6,26,27}$. For STED imaging, two different microscopes were used: (i) a Leica TCS STED microscope (Fig. 3d-g) using two pulsed lasers for excitation (diode laser, $635 \mathrm{~nm}$, 
$<90 \mathrm{ps}$ ) and stimulated emission (Ti:sapphire, $750 \mathrm{~nm}, \sim 300 \mathrm{ps}$ ), with both lasers running at a repetition rate of $80 \mathrm{MHz}$ and synchronized to each other to ensure optimal STED efficiency in the focal plane; and (ii) a custom microscope ${ }^{50}$ (Fig. $\left.3 \mathbf{h}-\mathbf{j}\right)$. This microscope used identical lasers but allowed for higher STED powers and therefore exhibited a resolution of $\sim 50 \mathrm{~nm}$ and $\sim 150 \mathrm{~nm}$ for two-dimensional $(x, y)$ and three-dimensional $(x, y, z)$ imaging, respectively. Single confocal and STED images of ribbons and $\mathrm{Ca}^{2+}$ channel clusters were acquired after adjusting the focus of the $\times 100$ oil immersion lens $(\mathrm{NA}=0.7$ or 1.4$)$ to the fluorescence maximum of an object of interest, as found in a $x z$-scan.

\section{Electron microscopy. See Supplementary Methods.}

Data analysis. Data was analyzed using Matlab (MathWorks), Igor Pro (Wavemetrics) and ImageJ software. Figures were assembled for display in Adobe Photoshop and Illustrator software. Mean $\Delta C_{\mathrm{m}}$ and $\mathrm{Ca}^{2+}$ current estimates are grand means calculated from the mean estimates of individual IHCs. Means were expressed \pm s.d. (or s.e.m. when noted). If applicable (that is, as determined by normality of the distribution (Jarque-Bera test) and equal variances between ( $F$-test) the two samples), an unpaired, two-tailed $t$-test was used to compare the two samples. In all other cases, a Mann-WhitneyWilcoxon test was used.

Image analysis. For synaptic cochleograms, CtBP2/RIBEYE immunofluorescence spots in the basolateral portion of IHCs (up to the apical end of the CtBP2-stained nucleus) were counted in $z$-stacks and divided by the number of IHCs (taken as the quantity of nuclei in the field of view). Estimation of the cellular synapse distribution was performed using custom-written MATLAB routines (available at http://www.innerearlab.uni-goettingen.de/) that included (i) alignment of the image stacks with the tonotopic axis, (ii) image segmentation into ribbons (positive, if more than four connected voxels were above threshold) and nuclei, (iii) center of mass calculation for both types of structures (by a three-dimensional gaussian fit in the case of nuclei), (iv) assignment of ribbons to the closest IHC nucleus, (v) alignment of the individual IHC's $z$-axis to a common $z$-axis, (vi) normalization of the cell's $z$-extent (measured from the center of the nucleus to the basal end of the IHC) to the population average and (vii) vector calculation. For Figure $\mathbf{2} \mathbf{b}$, we also normalized the radial extent of the IHCs for improved superposition. The FWHM in confocal and STED images was estimated using gaussian functions (two-dimensional for morphology, one-dimensional for functional imaging). The two-dimensional data in Figure $\mathbf{3} \mathbf{j}$ was linearly deconvolved with a two-dimensional gaussian PSF (FWHM of $70 \mathrm{~nm}$ for the STED and $250 \mathrm{~nm}$ for the confocal).

Note: Supplementary information is available on the Nature Neuroscience website.

\section{ACKNOWLEDGMENTS}

We thank E. Neher, M. Göpfert, R. Nouvian, N. Strenzke, M. Müller and A. Lysakowski for comments on the manuscript; K. Wadel and C. Henrich for participation in an early stage of the project; A. Neef for image analysis support; J. Hegerman and S. Eimer for support with high-pressure rapid freeze and freeze substitution; and C. Rüdiger and M. Köppler for technical assistance. This work was supported by grants from the Deutsche Forschungsgemeinschaft (Center for Molecular Physiology of the Brain; T.M., A.E. and S.W.H), a Lichtenberg Fellowship from the state of Lower Saxony (T.F.), the European Commission (Eurohear, T.M.), the Max-Planck-Society (Tandemproject, T.M.), BMBF (Bernstein Center for Computational Neuroscience Göttingen, T.M.) and an intramural grant from the University of Göttingen Medical School (A.C.M.).

\section{AUTHOR CONTRIBUTIONS}

The study was designed by T.M., A.C.M., A.E. and T.F. The experimental work was performed by A.C.M., T.F., D.K., D.R., G.H., N.M.C., Y.M.Y. and B.H. S.W.H. co-developed the super-resolution microscopes.

Published online at http://www.nature.com/natureneuroscience/ Reprints and permissions information is available online at http://npg.nature.com/ reprintsandpermissions/

1. Fuchs, P.A. Time and intensity coding at the hair cell's ribbon synapse. J. Physiol. 566, 7-12 (2005).
2. Moser, T., Neef, A. \& Khimich, D. Mechanisms underlying the temporal precision of sound coding at the inner hair cell ribbon synapse. J. Physiol. (Lond.) 576, 55-62 (2006).

3. Nouvian, R., Beutner, D., Parsons, T.D. \& Moser, T. Structure and function of the hair cell ribbon synapse. J. Membr. Biol. 209, 153-165 (2006).

4. Sterling, P. \& Matthews, G. Structure and function of ribbon synapses. Trends Neurosci. 28, 20-29 (2005).

5. Moser, T. \& Beutner, D. Kinetics of exocytosis and endocytosis at the cochlear inner hair cell afferent synapse of the mouse. Proc. Natl. Acad. Sci. USA 97, 883-888 (2000).

6. Khimich, D. et al. Hair cell synaptic ribbons are essential for synchronous auditory signalling. Nature 434, 889-894 (2005).

7. Griesinger, C.B., Richards, C.D. \& Ashmore, J.F. Fast vesicle replenishment allows indefatigable signalling at the first auditory synapse. Nature 435, 212-215 (2005).

8. Goutman, J.D. \& Glowatzki, E. Time course and calcium dependence of transmitter release at a single ribbon synapse. Proc. Natl. Acad. Sci. USA 104, 16341-16346 (2007).

9. Platzer, J. et al. Congenital deafness and sinoatrial node dysfunction in mice lacking class D L-type Ca2+ channels. Cell 102, 89-97 (2000).

10. Brandt, A., Striessnig, J. \& Moser, T. CaV1.3 channels are essential for development and presynaptic activity of cochlear inner hair cells. J. Neurosci. 23, 10832-10840 (2003).

11. Brandt, A., Khimich, D. \& Moser, T. Few CaV1.3 channels regulate the exocytosis of a synaptic vesicle at the hair cell ribbon synapse. J. Neurosci. 25, 11577-11585 (2005).

12. Matsubara, A., Laake, J.H., Davanger, S., Usami, S. \& Ottersen, O.P. Organization of AMPA receptor subunits at a glutamate synapse: a quantitative immunogold analysis of hair cell synapses in the rat organ of Corti. J. Neurosci. 16, 4457-4467 (1996).

13. Glowatzki, E. \& Fuchs, P.A. Transmitter release at the hair cell ribbon synapse. Nat. Neurosci. 5, 147-154 (2002).

14. Liberman, M.C. Morphological differences among radial afferent fibers in the cat cochlea: an electron-microscopic study of serial sections. Hear. Res. 3, 45-63 (1980).

15. Rutherford, M.A. \& Roberts, W.M. Frequency selectivity of synaptic exocytosis in frog saccular hair cells. Proc. Natl. Acad. Sci. USA (2006).

16. Johnson, S.L., Forge, A., Knipper, M., Munkner, S. \& Marcotti, W. Tonotopic variation in the calcium dependence of neurotransmitter release and vesicle pool replenishment at mammalian auditory ribbon synapses. J. Neurosci. 28, 7670-7678 (2008).

17. Liberman, M.C. The cochlear frequency map for the cat: labeling auditory-nerve fibers of known characteristic frequency. J. Acoust. Soc. Am. 72, 1441-1449 (1982).

18. Muller, M., von Hunerbein, K., Hoidis, S. \& Smolders, J.W. A physiological placefrequency map of the cochlea in the CBA/J mouse. Hear. Res. 202, 63-73 (2005).

19. Liberman, M.C., Dodds, L.W. \& Pierce, S. Afferent and efferent innervation of the cat cochlea: quantitative analysis with light and electron microscopy. J. Comp. Neurol. 301, 443-460 (1990); erratum 304, 341 (1991).

20. Slepecky, N.B., Galsky, M.D., Swartzentruber-Martin, H. \& Savage, J. Study of afferent nerve terminals and fibers in the gerbil cochlea: distribution by size. Hear. Res. 144 124-134 (2000).

21. Francis, H.W., Rivas, A., Lehar, M. \& Ryugo, D.K. Two types of afferent terminals innervate cochlear inner hair cells in C57BL/6J mice. Brain Res. 1016, 182-194 (2004).

22. Liberman, M.C. Single-neuron labeling in the cat auditory nerve. Science 216, 1239-1241 (1982).

23. Taberner, A.M. \& Liberman, M.C. Response properties of single auditory nerve fibers in the mouse. J. Neurophysiol. 93, 557-569 (2005)

24. Merchan-Perez, A. \& Liberman, M.C. Ultrastructural differences among afferent synapses on cochlear hair cells: correlations with spontaneous discharge rate. J. Comp. Neurol. 371, 208-221 (1996).

25. Ruel, J. et al. Dopamine inhibition of auditory nerve activity in the adult mammalian cochlea. Eur. J. Neurosci. 14, 977-986 (2001).

26. Hell, S. \& Stelzer, E.H.K. Properties of a 4Pi-confocal fluorescence microscope. J. Opt. Soc. Am. A 9, 2159-2166 (1992)

27. Egner, A., Jakobs, S. \& Hell, S.W. Fast 100-nm resolution three-dimensional microscope reveals structural plasticity of mitochondria in live yeast. Proc. Natl. Acad. Sci. USA 99, 3370-3375 (2002).

28. Hell, S.W. \& Wichmann, J. Breaking the diffraction resolution limit by stimulated emission: stimulated emission depletion microscopy. Opt. Lett. 19, 780-782 (1994).

29. Muller, M. The cochlear place-frequency map of the adult and developing Mongolian gerbil. Hear. Res. 94, 148-156 (1996).

30. Wu, Y.C., Tucker, T. \& Fettiplace, R. A theoretical study of calcium microdomains in turtle hair cells. Biophys. J. 71, 2256-2275 (1996).

31. Martinez-Dunst, C., Michaels, R.L. \& Fuchs, P.A. Release sites and calcium channels in hair cells of the chick's cochlea. J. Neurosci. 17, 9133-9144 (1997).

32. Masugi-Tokita, M. et al. Number and density of AMPA receptors in individual synapses in the rat cerebellum as revealed by SDS-digested freeze-fracture replica labeling. J. Neurosci. 27, 2135-2144 (2007).

33. Tokuoka, H. \& Goda, Y. Activity-dependent coordination of presynaptic release probability and postsynaptic GluR2 abundance at single synapses. Proc. Natl. Acad. Sci. USA 105, 14656-14661 (2008).

34. Rostaing, P., Weimer, R.M., Jorgensen, E.M., Triller, A. \& Bessereau, J.L. Preservation of immunoreactivity and fine structure of adult $C$. elegans tissues using high-pressure freezing. J. Histochem. Cytochem. 52, 1-12 (2004).

35. Roberts, W.M., Jacobs, R.A. \& Hudspeth, A.J. Colocalization of ion channels involved in frequency selectivity and synaptic transmission at presynaptic active zones of hair cells. J. Neurosci. 10, 3664-3684 (1990).

36. Tucker, T. \& Fettiplace, R. Confocal imaging of calcium microdomains and calcium extrusion in turtle hair cells. Neuron 15, 1323-1335 (1995). 
37. Schnee, M.E., Lawton, D.M., Furness, D.N., Benke, T.A. \& Ricci, A.J. Auditory hair cellafferent fiber synapses are specialized to operate at their best frequencies. Neuron 47, 243-254 (2005)

38. Beutner, D., Voets, T., Neher, E. \& Moser, T. Calcium dependence of exocytosis and endocytosis at the cochlear inner hair cell afferent synapse. Neuron 29, 681-690 (2001).

39. Rodriguez-Contreras, A. \& Yamoah, E.N. Direct measurement of single-channel $\mathrm{Ca}^{2+}$ currents in bullfrog hair cells reveals two distinct channel subtypes. J. Physiol. (Lond.) 534, 669-689 (2001).

40. Frank, T., Khimich, D., Neef, A. \& Moser, T. Mechanisms contributing to synaptic $\mathrm{Ca}^{2+}$ signals and their heterogeneity in hair cells. Proc. Natl. Acad. Sci. USA (in the press).

41. Issa, N.P. \& Hudspeth, A.J. Clustering of $\mathrm{Ca}^{2+}$ channels and $\mathrm{Ca}^{2+}$-activated $\mathrm{K}^{+}$channels at fluorescently labeled presynaptic active zones of hair cells. Proc. Natl. Acad. Sci. USA 91, 7578-7582 (1994).

42. Zenisek, D., Davila, V., Wan, L. \& Almers, W. Imaging calcium entry sites and ribbon structures in two presynaptic cells. J. Neurosci. 23, 2538-2548 (2003).
43. Tsuji, J. \& Liberman, M.C. Intracellular labeling of auditory nerve fibers in guinea pig: central and peripheral projections. J. Comp. Neurol. 381, 188-202 (1997).

44. Ehret, G. Age-dependent hearing loss in normal hearing mice. Naturwissenschaften $\mathbf{6 1}$, 506-507 (1974).

45. Liberman, M.C. et al. Prestin is required for electromotility of the outer hair cell and for the cochlear amplifier. Nature 419, 300-304 (2002).

46. Ehret, G. Common rules of communication sound perception. in Behavior and Neurodynamics for Auditory Communication (ed. J.S. Kanwal \& G. Ehret) 85-114 (Cambridge University Press, Cambridge, 2006).

47. Fuchs, P.A., Glowatzki, E. \& Moser, T. The afferent synapse of cochlear hair cells. Curr. Opin. Neurobiol. 13, 452-458 (2003).

48. Nusser, Z. AMPA and NMDA receptors: similarities and differences in their synaptic distribution. Curr. Opin. Neurobiol. 10, 337-341 (2000).

49. Johnson, S.L. \& Marcotti, W. Biophysical properties of CaV1.3 calcium channels in gerbil inner hair cells. J. Physiol. (Lond.) 586, 1029-1042 (2008).

50. Harke, B. et al. Resolution scaling in STED microscopy. Opt. Express 16, 4154-4162 (2008). 